\title{
Telemedicine Effects: Cost, Quality, and Access
}

\author{
Rashid L. Bashshur
}

\begin{abstract}
Following a brief review of lessons learned from first generation telemedicine projects, an analytical framework for assessing the potential effects of telemedicine on cost, quality, and accessibility of health care is provided. It is proposed that the effects of telemedicine on cost, quality, and accessibility are interconnected, and a comprehensive assessment should incorporate all three aspects, each considered from the perspectives of clients, providers, and society.
\end{abstract}

\section{INTRODUCTION}

One of the most critical policy concerns with regard to the development of telemedicine is whether or not the dependence on telecommunications and computer technology for the delivery of health care will increase or decrease its overall cost. The emphasis on cost is necessary because the introduction of a new technology is usually accompanied by questions about its potential inflationary effect. Historically, the use of expensive technology has contributed to the total increase in the cost of care.

The major purpose of this paper is to provide a contextual and conceptual framework for the analysis of the potential effects of telemedicine on the health care system with special emphasis on cost. However, while cost is certainly important, it cannot and should not be viewed independently. Conceptually, it must be viewed in conjunction with the simultaneous impact of telemedicine on the accessibility and quality of medical care. Contextually, we must examine the past for pertinent lessons learned. Therefore, this paper will review briefly the results of the experience with telemedicine to date, describe the framework for a valid assessment of telemedicine effects on the health care system, discuss the rationale concerning the expected telemedicine effects on cost, quality, and

From the Department of Health Management and Policy, School of Public Health, The University of Michigan, Ann Arbor, Michigan 48109.

Paper presented at the Second National Aeronautics and Space Administration/Uniformed Services University of the Health Sciences, International Conference on Telemedicine, Bethesda, Maryland, September 8-10, 1994. 
accessibility to care, and propose ways to resolve telemedicine issues related to cost, access, and quality.

In considering the potential merit of telemedicine, it is important to focus on the following points. First, the dearth of systematic empirical research regarding the true effects of telemedicine on the cost, quality and accessibility of care has left the field ripe for speculation and opinion. Much of this opinion is counterpoised. The majority suggests that telemedicine will improve accessibility, enhance quality, and contain cost. This view considers telemedicine as a systematic innovation to redress a broad spectrum of health care problems, including the geographic maldistribution of health care resources, the uneven distribution of quality, and the spiralling cost of care. A minority has cautioned about a potential for decreased accessibility and quality and increased costs. The latter viewpoint, while conceding that telemedicine is a potentially beneficial innovation, nonetheless expresses concern about an increase in the overall cost of care as a result of increased demand for service, as well as the displacement of rural providers and possible abuse. Such an increase, however, should it materialize, may very well represent a pent-up demand or an unmet need for care. Without solid research, and especially studies that consider the interactions of cost, access, and quality of care, we cannot reach closure on these issues.

Second, in view of the intensity of the current interest in telemedicine as a technologically-based response to some of the most intractable problems in health care delivery, it is imperative that we develop a clear understanding of the role of telemedicine in the health care system and the reasons underlying the expectations of its potential for the resolution of these problems. The basic technology that drives telemedicine has developed a life of its own as part of the information infrastructure for the nation. This technology is being, and will be, used in an increasing variety of human services and businesses. Typically, the trend in the adoption of new technology is irreversible especially after its usefulness has been demonstrated, regardless of cost. However, the extent to which the technological imperative applies to telemedicine implementation remains to be seen.

Third, due to the confluence of several historical trends, we are faced with a window of opportunity to maximize the return on the investment in telemedicine by designing optimal systems which match the specific needs of target populations and communities. In order to do this, though, we must start with a clear definition of telemedicine. Briefly, we can define telemedicine as a system of care that uses telecommunications and computer technology to substitute for face-to-face interaction between patients, physicians, and/or non-physician providers in various combinations. However, there is no consensus to date on this or other definitions. We need to reach agreement as to whether telemedicine is a new system of delivering medical care or simply a technological augmentation to existing arrangements. If new, what are its unique attributes and capabilities? What are the structural requirements for optimal telemedicine systems that are likely to maximize the beneficial effects in health care delivery while minimizing undesirable effects? Answers to such questions are fundamental for understanding the nature of telemedicine's input to the health care process and for designing appropriate studies to assess its outcomes. That is to say, we should start with a clear understanding of what we mean by telemedicine and telemedicine systems, and the specific ways in that telemedicine can address serious problems in health care delivery. From there, effective systems to achieve these objectives can be developed and subsequently evaluated. 


\section{The First Generation of Telemedicine}

Before discussing the framework for analyzing the potential effects of telemedicine, it may be helpful to review the experience with telemedicine and what we have learned to date.

Telemedicine has reemerged in force in health care after a short-lived but auspicious beginning in the early 1970s. The first generation of telemedicine projects was supported by the former Department of Health, Education, and Welfare (now Health and Human Services), the National Science Foundation, the former Office of Economic Opportunity, and the National Aeronautics and Space Administration. Though much was learned from these projects, they were discontinued just as the debate about telemedicine reached a critical stage and before important questions about cost, access, and quality could be answered. Proponents were advocating its universal implementation as a means to improve health care delivery at an affordable price and with minimal disruption to the existing system of care. Yet, despite the promise of ameliorating these problems, the entire first generation of telemedicine projects was prematurely terminated in a cloud of doubt. Their demise was not the result of any empirical findings depicting their failure to achieve stated objectives. Rather, these projects ended without reaching closure on the major questions regarding their merit. Major reasons for their demise include: (a) people's unfamiliarity and limited experience with them; (b) the absence of institutional commitments for sustaining them beyond the initial funding from external sources and the absence of incentives for physicians; (c) limitations in the technology of the time; and (d) poor planning and design, which adversely affected the stability of the systems.

At that time, much like today, there were strong advocates and a few critics who assumed opposite sides of the telemedicine question. If history is not to be repeated, important lessons must be drawn from the first generation of telemedicine. More importantly, understanding these lessons should help us maximize the benefits accruing from telemedicine systems, guide us in conducting current assessments of telemedicine, and help us avoid such egregious errors as prematurely dismissing telemedicine once again without evidence to support its rejection. However, there are already disturbing signs of just such shortsightedness. For instance, The Bureau of National Affairs quoted a preliminary draft of a recent report commissioned by the Health Care Financing Administration (HCFA) that "telemedicine may not be cost-effective . . . and . . . that telemedicine increases the potential for fraud and abuse of the system." There is no evidence whatsoever to support these claims. To be able to claim cost-ineffectiveness, the cost of delivering a given set of services with telemedicine should be greater than by conventional means. No such analyses have been done on any systematic basis. Similarly, the claim that telemedicine increases the potential for fraud and abuse can only be justified if there is evidence of an independent telemedicine effect in this regard. No such evidence exists. In fact, it can be argued more logically that telemedicine should decrease the potential for fraud and abuse. Clinical consults typically require the co-presence of two providers with the patient. In effect, this produces a "built-in" second opinion feature that should reduce the likelihood of unnecessary and inappropriate service. Also, most telemedicine consults are recorded on video tape which become part of patients' records. This provides explicit evidence of any malfeasance or nonfeasance on the part of providers. Finally, so far, no telemedicine network has been established to enrich its developers and providers by 
referring patients to their own company for financial gain. Even if this happens, an independent telemedicine effect has to be confirmed to ascertain telemedicine as the culprit. To date, the use of telemedicine systems nationwide has been rather low.

What are the important lessons from the first generation of telemedicine projects? Overall, we can say that technology was constrained in the early projects, especially in terms of transmission-relay, audio quality, and equipment reliability. Today, however, the questions regarding feasibility and clinical effectiveness of telemedicine have been largely put to rest. Research has demonstrated the clinical feasibility and relative effectiveness of specific clinical services, such as telepsychiatry, ${ }^{2-4}$ teledermatology, ${ }^{5,6}$ telecardiology, ${ }^{7}$ and teleanesthesia. ${ }^{8}$ The effectiveness of diagnostic technology in radiology and pathology has also been demonstrated, with the caveat that for the latter, diagnostic effectiveness is a function of specific technologies and site of pathology. ${ }^{9}$

In addition, the following specific observations can be made:

1. None of the first generation telemedicine systems was able to achieve its real or potential market niche. Hence, we have yet to determine the nature and size of the demand for telemedicine services. Perhaps it was unrealistic to expect that the initial projects would operate at full capacity or would achieve their full market potential. Nevertheless, a realistic assessment of cost-benefit relationships would have required that these experimental systems achieve a steady state of operation.

2. With minor exceptions, no serious contextual or situational analysis was conducted to establish a logical fit between the characteristics of the environment and the types of systems that were put in place. Hence, it was not clear what specific needs were to be met by each telemedicine project. Without such definition, there was no way to determine the extent of their success or failure. Moreover, no attempt was made to determine the organizational attributes necessary to sustain telemedicine systems.

3. The vast majority of telemedicine projects focused on a limited set of clinical functions, thereby restricting the potential utility of these systems for a broad spectrum of clinical and educational functions for providers and clients. More critically, no attempt was made to exploit the integrative capacity of the technology of telemedicine to support, and thereby improve, the efficiency and productivity of existing health care systems.

4. Even though interest in these issues was very strong, there was little empirical research directed toward the role of telemedicine in the health care delivery system. For instance, Murphy and Bird ${ }^{10}$ referred to telemedicine as "a new community health resource." But, aside from the statement's intuitive appeal, these authors did not describe clearly how this would work or provide specific evidence as to how this resource would be utilized in the community.

5. Finally, and most importantly for the present discussion, there was no serious or competent analysis of the cost and benefits/effects of telemedicine that can serve as a valid basis for policy determination. The capital and operational costs were substantial for first generation telemedicine systems, less so for the second generation. Hence, without a full exploitation of the capability of these systems, their cost appeared excessive. A logical question was raised at the time as to whether lower-cost technology could be more fully and, therefore, more cost-effectively utilized. But even that question has yet to be answered.

Some additional unanswered questions from the first generation of telemedicine projects regarding bandwidth, relay, resolution, and switching, and computer interface 
have been largely obviated by advances in the technology. Indeed, earlier studies which revealed limitations in certain aspects of teleradiology, telepathology, and the electronic stethoscope simply reflected limitations in the technology of the period, as mentioned earlier. Some questions about provider and client acceptance also reflected, to a certain extent, remediable conditions such as equipment breakdown, flawed system design, and inefficient location.

In summary, while past research provides useful information about the clinical feasibility and effectiveness of specific clinical services, it has not addressed the broader questions concerning the effects of telemedicine on the health care system. Consequently, the expectation of telemedicine engendered in the early 1970 s as a solution to the multifaceted and seemingly intractable problems of high cost and uneven quality of care, health resource maldistribution, and limited accessibility to care for some people, has not been realized. ${ }^{11}$ As we enter a new era in the development of telemedicine, we need to develop a clear perspective on the concept of telemedicine and its specific role in the health care system.

\section{The Promise of Telemedicine}

It would be much more helpful for this audience to present definitive empirical findings rather than speculation concerning the observed impact of telemedicine on health care delivery. Our knowledge of telemedicine's true effects lags far behind the current rush to establish telemedicine systems in various parts of the country. Despite its designation as a second generation, telemedicine is still in infancy. Serious empirical research and evaluation are in gestation. Consequently, it will be some time before we have any hard data to serve as a valid foundation for theoretically-based and policy-relevant empirical analysis.

Nonetheless, the future of telemedicine is being shaped today conceptually, contextually, and concretely. Before too long, the basic technology will become ubiquitous in health care as in other human services and businesses. Our discussions and the decisions we make today regarding telemedicine will shape its future for years to come. These include the conceptual definition of telemedicine we adopt, the environmental contexts in which we implement telemedicine, the specific system configurations we put in place (including combinations of technology, manpower, organization and clinical services), as well as the kind of information we gather and research we conduct. Indeed, what we are offered today is a unique opportunity to develop a systematic agenda for telemedicine research which will, in turn, provide the needed scientific knowledge base. Furthermore, with this agenda, we have the opportunity to address the critical policy issues surrounding telemedicine's effects on the health care system.

At the risk of oversimplification, it may be suggested that all the potential benefits of telemedicine derive from the substitutions made possible by telecommunications and computer technology. These substitutions include site of care, type of provider rendering services, and the content and process of care. Basically, the technology permits the interaction and the exchange of information between geographically remote interactants as a substitute for face-to-face contact. Whether such interaction occurs between physician and physician; patient and physician; or non-physician provider, patient and physician, the basic technology enables those involved in these clinical encounters to conduct their 
business without face-to-face contact. Diagnostic procedures, clinical consultations, and continuing medical education can successfully be carried out using telemedicine without seriously compromising the quality of the information exchanged, effectiveness of education, or the safety of the patient being treated. In many instances, the quality of information, education, and patient safety may be enhanced.

The intrinsic merit of telemedicine derives from the ability to distribute and control the use of medical service for maximal health effects. The control of use of service should occur in a rational process whereby appropriate use of service is encouraged (by diminishing or removing distance and time barriers) while discouraging inappropriate use (by maintaining reasonable financial disincentives). As well, it should encourage the use of a more appropriate mix of providers (physician assistant, nurse practitioner, primary care physician, and/or specialist) depending on patients' needs. These services should be provided at an appropriate site which is convenient for both patients and providers. Like any other system, telemedicine can be put to good or bad use. Its good use is a function of rational substitutions, as will be described later.

\section{Framework for Assessment}

The potential effects of telemedicine may be assessed categorically or comprehensively. The categoric approach focuses on specific sets of effects, whereas the comprehensive approach encompasses a variety of effects from a variety of perspectives simultaneously. The comprehensive approach is demonstrated here because it provides a more realistic contextual and analytical basis for the assessment of telemedicine effects and the subsequent determination of the interactions between the different effects. For example, a comprehensive approach enables the analysis of the relationship between improved access and cost, as well as the tradeoffs between cost and quality. This is represented schematically by a matrix (Figure 1) that depicts essential perspectives on one axis and types of effects on the other. Using this matrix, we can examine the specific as well as the interactive effects of telemedicine on accessibility, cost, and quality from the perspectives of clients, providers, and society at large. In the next sections of this paper, the three potential effects of telemedicine and their interactions will be identified and described.

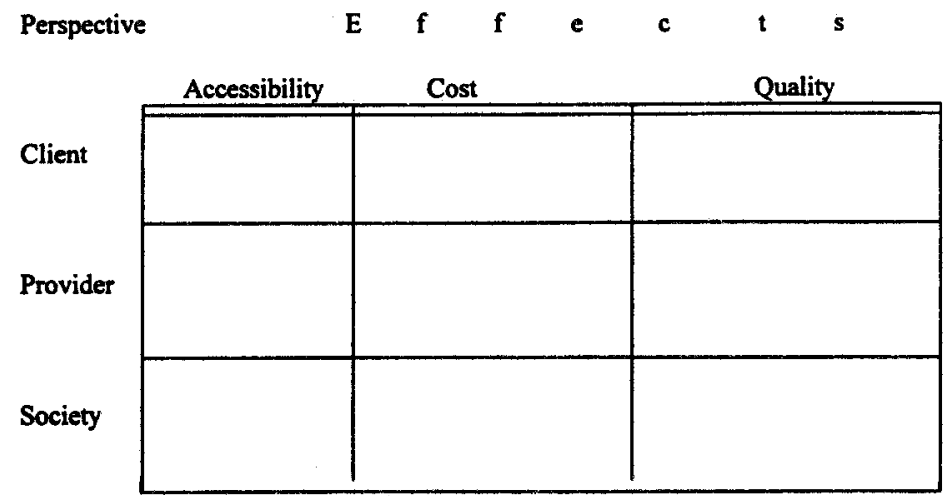

Figure 1. Matrix for analysis of systemic effects of telemedicine. 


\section{Accessibility Effects}

Accessibility is a multidimensional concept. Within the context of health care, it refers to the relative ease or difficulty in obtaining health services. Operationally, from the client's perspective, accessibility is defined in terms of the extent to which they face geographic, economic, architectural, cultural, and/or social barriers to needed care. Much of the recent attention on accessibility to care has focused on the uninsured and underinsured. However, financial factors constitute a necessary but not sufficient base for gaining access to care. ${ }^{12}$ When financial barriers are removed, geographic and other factors become more prominent. ${ }^{13}$

The target populations and the major beneficiaries of telemedicine are the geographically remote, the institutionally confined, and those otherwise medically underserved, including inner city residents and the elderly. The substitution of telemedicine for personto-person encounters should reduce the need for travel and the related opportunity costs and other inconveniences encountered in the process of obtaining care. Instead of having to travel to distant tertiary care centers for specialized, and sometimes even routine, services, residents of rural areas, correctional institutions, and nursing homes could receive an array of services via telemedicine. Only when it is determined appropriate through consultation with specialists would it be necessary for clients to be referred or transferred to be served at the medical centers.

Telemedicine enables clients and providers to bridge the distance and time barriers that separate them. Obviously, these savings accrue primarily to clients. Since significant travel distance and time barriers exist for a substantial portion of the remote and confined populations, the hypothesis to be tested is the extent to which telemedicine reduces barriers and permits more appropriate use of medical care. However, desirable as these benefits are, there is a concern about the potential for encouraging inappropriate utilization by clients. All reductions in price, including time price, can create the so-called "moral hazard." This term refers to the increase in the demand for care that results from the reduction or removal of some barrier. Under such conditions, clients may be more likely to use services because they are less sensitive to price or other constraints. Research must be designed to capture and assess the extent of this potential effect as well.

Accessibility for providers in both remote and central sites relates to convenience, opportunity cost, and work load. Providers located in remote and isolated areas and institutions will have ready access to consultants and referral sources. With telemedicine, they may encounter less "red tape" in arranging for both consultations and referrals. Remote providers may be able to alleviate their work load and coverage during off-hours because of their link to medical centers and the use of non-physician providers. Providers located at tertiary care centers will be able to offer their expertise to a much larger and diverse provider and client population compared to those only seen at their medical centers. Forthcoming distributive technology, which includes multimedia desktop computer terminals, would further reduce barriers for providers and enable them to provide telemedicine consultations without leaving their offices or clinics.

From a societal perspective, improved access to care may increase the overall level of satisfaction with life in the rural community. In some instances, this may lead to greater community stability. On the other hand, reduced distance and time barriers to care may also create other expectations which are more difficult to fulfill. For example, will the 
enhanced convenience of telemedicine in terms of reduced travel produce equivalent expectations in convenience for appointment, waiting, and/or service times? If the new expectations are not met, how will the clients feel about the discrepancy? In an earlier well-designed study, telemedicine service time was found to be longer than face-to-face encounters. ${ }^{14}$ One task for research in this regard is the determination of the relationship between client expectations and the abilities of the providers and the system to meet them.

Finally, it should be clarified that one of the most significant interactions occurs between accessibility and cost, as mentioned earlier. If time and distance barriers to care are removed, use of service is likely to increase. An increase in volume without a commensurate decline in price will increase total cost. But here again, increased demand for care must be examined in relation to the level of need in the population and subsequent effects on health.

\section{Cost Effects}

Economic analysis entails a comparison of specified sets of inputs and outputs. This requires a precise definition of system characteristics in terms of quantitative and qualitative capability. Once the definition is made, economic analysis may be conducted in several different ways, each answering a different question pertaining to cost. The predominant methods are cost-effectiveness analysis (CEA) and cost-benefit analysis (CBA). Cost-benefit analysis (CBA) uses standardized measures (in monetary terms) to compare costs and benefits. Cost-effectiveness analysis (CEA) compares costs of rendering a standardized set of services by alternative methods, i.e., it compares costs with some measure of effectiveness. The critical components of CBA/CEA analysis are (a) the specification of desired outputs, and (b) a clear definition of the intervention or sets of inputs, and costs.

The most common questions in telemedicine, as in all new technologies or arrangements in health care delivery, are stated in terms of costs and effects or benefits. Since economic analysis, generically, focuses on comparing specified sets of inputs and outputs in different systems, in the case of telemedicine, the comparison should be made between telemedicine and alternative arrangements. The result is a determination of comparative benefits or effects and costs under the alternative arrangements.

As students of health care organization have repeatedly emphasized, the primary objectives of the health care system are to promote health, minimize illness and disability, and ameliorate pain and suffering. If one accepts these objectives as the desired outputs of the health care system, economic analysis can then inform the policymaker regarding the least costly or most beneficial alternative to achieve them. Cost-effectiveness analysis will determine the least costly system that is capable of reaching these objectives. In CBA, on the other hand, a complete listing of all desired objectives are rank-ordered by least cost for their attainment in order to maximize the benefits for given levels of expenditure. Cost-benefit analysis will determine the various gains or losses (converted to monetary values) that result from telemedicine.

These two analytic frameworks are capable of determining the extent to which telemedicine is an economically prudent investment because they compare the costs, the production process, and the outputs of telemedicine and traditional alternatives in the delivery of care. It has been suggested that cost-effectiveness analysis may be an ineffi- 
cient choice because of the multiplicity of objectives that might be achieved by telemedicine. Thus, "the cost-effectiveness approach is always one of sub-optimization." 15 An important caution against heavy reliance on CBA/CEA is offered by Warner and Luce. ${ }^{16}$ They point out that such analyses not only "will not rectify the general problem of health resource misallocation" but also may exacerbate "the problems because [they] emphasize measurable costs and benefits, and consequently deemphasize considerations of equity and the like."

The cost of telemedicine can be also measured without reference to benefits or effectiveness. Different components of cost can be measured or estimated, such as capital and operational costs, production cost, and unit and total cost. Because funding for telemedicine systems may come from different sources, the distinction between capital and operational costs may be critical. Some programs may be able to receive grants or subsidies for equipment acquisition whereas operational expenses are borne by the organization. Operational costs may be fixed on the basis of dedicated transmission lines and maintenance contracts. Alternatively, they may be variable on the basis of actual usage.

Typically, telemedicine developers are concerned about the size of the investment in the technology and the operating expenses in relation to the return on revenues generated. However, it may be suggested that the economic viability of telemedicine may not be dependent on these costs, despite their obvious importance. This is because telemedicine may have the potential of addressing issues of accessibility, cost containment, and quality assurance at the same time. While this remains to be empirically demonstrated, if telemedicine can bring quality health services to isolated and underserved populations at a reasonable price, the benefits will far outweigh the initial capital investment as well as the cost of operations. In addition, telemedicine systems can be designed with built-in mechanisms for cost-containment. Formal triage, for example, can be used to control patients' entry into the care process and to direct care throughout their illness episodes. In addition, cost containment may be achieved by substituting lower cost providers and facilities for those costing more, a potential for decreased intensity of care, and possibly a reduced need for care.

\section{Quality Effects}

Concern with quality in health care has been increasing as a result of rising costs and the need for accountability. Whereas quality has been measured in terms of structural, processual, and outcome indicators, ${ }^{17}$ greater emphasis is now placed on outcomes in order to ascertain the relationship between health care interventions and/or expenditures and health status. Two dimensions of quality are of special interest to telemedicine: technical and interpersonal. The technical dimension of quality pertains to the process of care and its outcome. The interpersonal dimension relates to personal treatment and satisfaction of both provider and client.

Improved quality of technical care for clients may be achieved from the potential for increased continuity and coordination of care, a "built-in" second opinion, the ready availability of specialist consultation upon demand, and the timeliness of care. Improvements in quality may also be achieved from reduced exposure to unnecessary diagnostic tests and procedures. 
Improved interpersonal quality may be achieved from greater satisfaction with the attention clients receive from their own physician or provider as a result of having more (or most) of their needs met locally rather than being inconvenienced by travel and relocation. On the other hand, the full range of attitudes regarding individual experience with telemedicine need to be explored. We need to learn about the extent of any feelings of apprehension or embarrassment and, if they exist, whether or not they change with greater familiarity with the technology. A related issue concerns the potential for perceptions of a two-class system of care, especially if telemedicine were perceived as less desirable than traditional practice.

From a provider perspective, the effects on quality may derive from two sources, one direct and the other indirect. The direct effect may result from the information transfer and the monitoring and control functions made possible by the telecommunications and computer technology. This is especially applicable to the isolated primary care provider in remote areas or places without ready access to specialist consultation. However, it may be appreciated that specialists in tertiary care centers may also benefit from a greater exposure to cases within their specialties. Since many of these specialists are involved in training physicians, they will gain a better appreciation of the actual conditions of practice outside of academic medical centers. Indirectly, providers in remote areas should overcome the effects of professional isolation and to keep abreast of technical advances in the science and practice of medicine.

A highly focused program for continuing medical education may be provided for rural physicians. It could use the patients and the typical conditions they encounter in their normal practice as teaching material, thereby enhancing the educational benefits derived from the continuing medical education.

Among the critical issues to be investigated in telemedicine practice is the conformity of primary care providers to prevailing professional standards of clinical practice in the diagnosis, treatment, and follow up of specific conditions and patients. The increased opportunity for professional interaction in telemedicine should enhance greater adherence to prevailing norms of clinical practice on the part of remote providers. However, this may well have the effect of increasing the intensity of care. Specialists may require more diagnostic tests and procedures in order to arrive at a definitive diagnosis. Under these conditions, quality may be improved and cost will also increase. Research must be attuned to picking up this potential interaction between quality and cost.

From a broader societal perspective the increased consistency in adherence to clinical norms of practice should tend to reduce the extant small area variations in utilization patterns. ${ }^{18}$ Subsequent reductions in excessively high rates of utilization for specified conditions in certain areas would also have the desirable effect of reducing the cost of care without jeopardizing quality.

Finally, it may be pointed out that the future of telemedicine may be secure, and its basic technology is here to stay and develop further. Much of the current interest in telemedicine is driven by a real concern to address some of the basic health care problems in the country. As demonstrated in this analysis, the effects of telemedicine are varied and complex, and there are real trade-offs and interactions among gains and losses. Such knowledge will become one of the essential inputs into the process of rational decision making and policy formulation regarding support for telemedicine. Hopefully, sound empirical research will answer the questions about all these issues and help us, ultimately, 
to design telemedicine systems in ways that bring maximal benefits to clients, providers, and society at large.

\section{REFERENCES}

1. Bureau of National Affairs. Health Care Pol. Rep. 2:26 (June 27), 1148-49, 1994.

2. Wittson C.L., and Benschoter, R.A., Two-way television: helping the medical center reach out. Am. J. Psychiatry 129:136-139, 1972.

3. Dwyer, T.F., Telepsychiatry: psychiatric consultation by interactive television. Am. J. Psychiatry 130: $865-869,1973$.

4. Dongier, M., Tempier, R., Lalinec-Michaud, M. and Meunier, D., Telepsychiatry: psychiatric consultation through two-way television. Can. J. Psychiatry 31:32-34, 1986.

5. Murphy, R.L.H., Fitzpatrick, T.B., Haynes, H.A., Bird, K.T., and Sheridan, T.B., Accuracy of dermatologic diagnosis by television. Arch. Derm. 105:833-835, 1972.

6. Rinde, E., Nordrum, I., and Nymo, B.J., Telemedicine in rural Norway. World Health Forum, 14:71-77, 1993.

7. Murphy, R.L.H., Block, P., and Bird, K.T., and Yurchak, P., Accuracy of cardiac auscultation by microwave. Chest 63:578-581, 1973.

8. Gravenstein, J.S., Berzima-Moettus, L., Regan, A., and Yoh-Han, B., Laser mediated telemedicine in anesthesia. Anesth. Analg. 53:605-608, 1974.

9. Cox, G.G., Cook, L.T., McMillan, J.H., Rosenthal, S.J., Dwyer, S.J., III, Chest radiography: comparison of high-resolution digital displays with conventional and digital film. Radiology 176:771-776, 1990.

10. Murphy, R.L.H., and Bird, K.T., Telediagnosis: A new community health resource. Observations and feasibility of telediagnosis based on 1000 patient transactions. Am. J. Public Health 64:113-119, 1974.

11. Bashshur, R.L., On the evaluation of telemedicine. Proceedings of the Office of Rural Health Policy Conference on Telemedicine, November 3-5, 1993, forthcoming.

12. Bashshur, R.L., Smith, D.G., and Stiles, R.A., Defining underinsurance: A conceptual framework for policy and empirical analysis. Med. Care Rev. 50:2 (Summer), 199-218, 1993.

13. Acton, J., Non-monetary factors in the demand for medical services: some empirical evidence. J. Polit. Econ. 83:595-614, 1975.

14. Moore, G.T., Willemain, T.R., Bonanno, R., Clark, W.D., Martin, A.R., and Mogielnicki, R.P., Comparison of television and telephone for remote medical consultation. N. Engl. J. Med. 292:729-732, 1975.

15. Berki, S.E., Telemedicine: some economic implications. Telemedicine: Explorations in the Use of Telecommunications in Health Care (R.L. Bashshur, et al., eds.), Charles C. Thomas, Springfield, Ill, 1975.

16. Wamer, K.E., and Luce, B.R., Cost-benefit and Cost-effectiveness Analysis in Health Care. Health Administration Press, Ann Arbor, 1982.

17. Donabedian, A., Explorations in Quality Assessment and Monitoring, Volume I: The Definition of Quality and Approaches to its Assessment, Health Administration Press, Ann Arbor, MI, 1982.

18. Wennberg, J., and Gittelsohn, A., Small area variations in health care delivery. Science 182:1102-1108, 1973. 